\title{
Design and Synthesis of aza-Bicyclononene Dienophiles for Rapid Fluorogenic Ligations
}

\author{
Sebastian J. Siegl, ${ }^{+}$Arcadio Vázquez, ${ }^{+}$Rastislav Dzijak, Martin Dračínský, Juraj Galeta, Robert \\ Rampmaier, Blanka Klepetářová, and Milan Vrabel ${ }^{*[a]}$
}

\begin{abstract}
Fluorogenic bioorthogonal reactions enable visualization of biomolecules under native conditions with excellent signal-tonoise ratio. Herein, we present the design and synthesis of conformationally-strained aziridine-fused trans-cyclooctene (azaTCO) dienophiles, which lead to the formation of fluorescent products in tetrazine ligations without the need for attachment of an extra fluorophore moiety. The presented aza-TCOs adopt the highly strained 'half-chair' conformation, which was predicted computationally and confirmed by NMR measurements and X-Ray crystallography. Kinetic studies revealed that the aza-TCOs belong to the most reactive dienophiles known to date. The potential of the newly developed aza-TCO probes for bioimaging applications is demonstrated by protein labeling experiments, imaging of cellular glycoconjugates and peptidoglycan imaging of live bacteria.
\end{abstract}

\section{Introduction}

Fluorogenic bioorthogonal reactions have emerged as a powerful tool for bioimaging as they offer an excellent signal-tonoise ratio without the need to use extensive washing steps. ${ }^{[1]}$ Significant progress has been made in the development of probes which can be activated by means of a selective chemical reaction in a complex biological environment. These include Staudinger ligation, ${ }^{[2]}$ azide-alkyne cycloadditions, ${ }^{[3]}$ lighttriggered nitrilimine-alkene cycloadditions ${ }^{[4]}$ and tetrazine ligations. ${ }^{[5]}$ In most cases, the fluorogenic probe contains an appropriately modified fluorophore moiety with its fluorescence being quenched via energy transfer and being restored upon the bioorthogonal chemical reaction. A much less common situation represents fluorogenic ligations where the formation of the fluorophore is an inherent result of the chemical reaction. One particular advantage of these types of probes is the absence of virtually any background fluorescence signal since no real prefluorophore (or any quenched fluorophore moiety) is present in the structure of reagents before the reaction takes place. One of the prominent examples of this type of reaction is the formation of pyrazolines upon cycloaddition of nitrilimines with alkenes. ${ }^{[6]}$ Shang et al. recently reported a conceptually similar styrenetetrazine cycloaddition leading to fluorescent dihydropyridazines. ${ }^{[7]}$ In parallel to the later study, we have shown that the inverse electron-demand Diels-Alder reaction

[a] MSc. S. J. Siegl, Dr. A. Vázquez, Dr. R. Dzijak, Dr. M. Dračínský, Dr. J. Galeta, MSc. R. Rampmaier, Dr. B. Klepetářová, Dr. M. Vrabel Department of Bioorganic and Medicinal Chemistry Institute of Organic Chemistry and Biochemistry of the Czech Academy of Sciences

Flemingovo nám. 2, 16610, Prague 6, Czech Republic E-mail:vrabel@uochb.cas.cz

$\left.{ }^{+}\right] \quad$ These authors contributed equally to this work

Supporting information for this article is given via a link at the end of the document.
(IEDDA) of 1,2,4,5-tetrazines with trans-cyclooctene (TCO) dienophiles can also directly lead to the formation of fluorescent products. In particular, we found that the axial trans-cyclooctene5-ol (ax-TCO-ol) leads upon reaction with various tetrazines to the formation of fluorescent dihydropyridazines with tuneable photophysical properties. ${ }^{[8]}$ Although we could demonstrate the power of this chemistry for bioimaging, we also found that by modifying the hydroxyl group of the ax-TCO-ol, the fluorogenic properties of the reaction alter or even completely disappear. For many reasons it would be advantageous to maintain the fluorogenic nature of the reaction while enabling attachment of various useful functional groups to e.g. biomolecules. The aim of the present study was to design new TCO derivatives, which would fulfil these criteria. Here, we show that by cis-fusion of an aziridine ring to the trans-cyclooctene skeleton a new type of highly reactive dienophiles are formed, which produce fluorescent products in IEDDA reaction with tetrazines. In addition, our kinetic measurements show that these strained alkenes belong to the most reactive bioorthogonal probes known to date. Their excellent reactivity is a result of the highly strained 'half-chair' conformation, which was confirmed by NMR measurements and, to the best of our knowledge, for the first time also by X-Ray crystallography.

\section{Results and Discussion}

\section{Rational design of aza-TCOs and computational methods}

Our previous in silico conformational analysis and density functional theory (DFT) modelling of transition state structures of the reaction between ax-TCO-ol and diphenyl-s-tetrazine showed that the hydroxyl oxygen atom of TCO is very close to one of the bridgehead hydrogen atoms in a low energy conformer of the IEDDA product. ${ }^{[8]}$ These calculations show that the conformation of the intermediate 4,5-dihydropyridazine promotes hydrogen migration to the hydroxyl oxygen, which results in the formation of the final 1,4-dihydropyridazine fluorophore. This means that the presence of a heteroatom in the right conformation is responsible for the rapid tautomerization of the dihydropyridazine and for the formation of fluorescent products. We therefore speculated that fusion of an aziridine ring to trans-cyclooctene would ensure the fluorogenicity of the reaction by introducing the required heteroatom in the right position. In addition, this should also force the trans-cyclooctene ring to adopt the highly strained 'half-chair' conformation which is known to enhance the rate of the IEDDA reaction dramatically. ${ }^{[9]}$ With this hypothesis in mind we performed a computational study with two aims: 1) to predict the most favourable conformation of aza-TCO compound 1a and 2) to predict the propensity of aza-TCO to form fluorescent products. The conformational analysis of compound 1a was performed with 100 molecular-mechanics simulated annealings. 
In 97 out of the 100 simulations, the resulting structure had the desired 'half-chair' conformation depicted in Figure 1A. The 'halfchair' conformation of $\mathbf{1 a}$ was also retained after geometry optimization by the DFT method (Figure $1 \mathrm{~A}$ and $\mathrm{ESI}$ ).

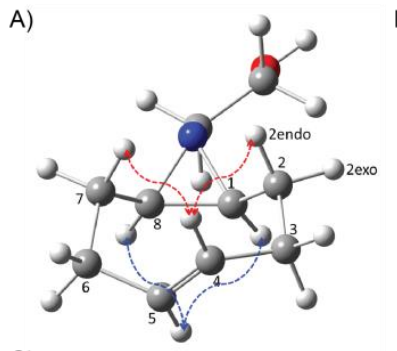

B)

\begin{tabular}{ccc} 
& Experimental & Calculated \\
\hline 2exo-3exo & 2.2 & 2.3 \\
6exo-7exo & 5.0 & 4.9 \\
1-2exo & 5.0 & 5.2 \\
8-7exo & 3.6 & 3.7 \\
4-3endo & 3.8 & 3.8 \\
5-6exo & 6.2 & 6.2
\end{tabular}

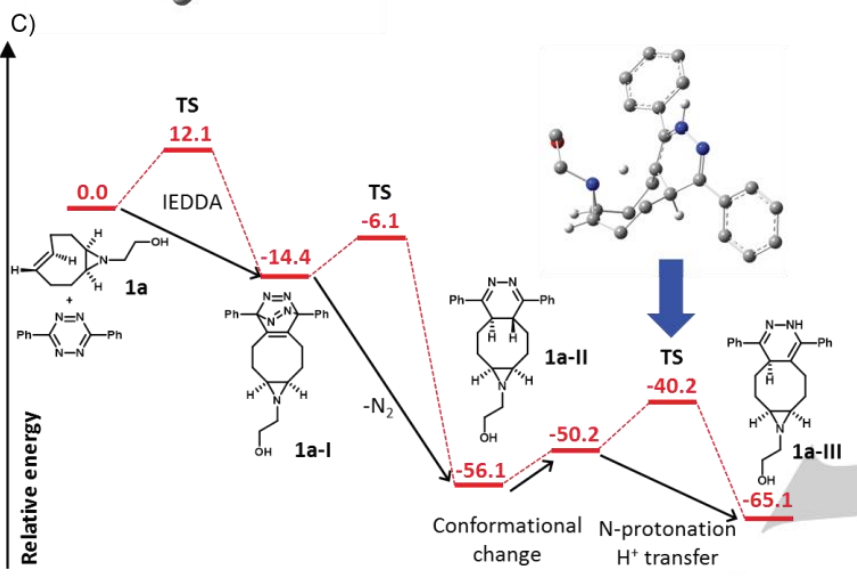

Figure 1. A) Lowest-energy conformation found by molecular modelling together with the most important experimental NOE contacts (indicated by arrows) confirming the half-chair conformation of 1a B) Selected experimental and calculated (B3LYP/6-31+g(d,p)/PCM) vicinal coupling constants $(\mathrm{Hz})$ between hydrogen atoms in 1 a $C$ ) The proposed reaction pathway for the reaction of $\mathbf{1 a}$ with diphenyl-s-tetrazine leading to fluorescent product $\mathbf{1 a - I I I}$ and the transitions-state structure.

The expected first step in the reaction sequence of compound 1a with 1,2,4,5-tetrazines is the IEDDA reaction followed by nitrogen elimination, which provides 4,5dihydropyridazine intermediate 1a-II (Figure 1C). The transition state structures were predicted by DFT computations for both of these reactions starting from compound 1a and diphenyl-stetrazine and the energy barriers toward intermediates 1a-I and 1a-Il were found to be 12.1 and $8.3 \mathrm{kcal} / \mathrm{mol}$, respectively. The key reaction step found previously for the formation of a 1,4dihydropyridazine fluorophore involved a hydrogen atom transfer from one of the bridgehead carbon atoms to a heteroatom. This hydrogen atom transfer was only possible for conformations with short distances between the hydrogen atom and the heteroatom. Therefore, we performed conformational analysis of compound 1a-II by simulated annealing followed by DFT geometry optimization, which revealed that a low-energy conformer of 1aII has the bridgehead hydrogen-aziridine nitrogen distance of $2.29 \AA$, which is suitable for the hydrogen atom migration to take place. The transition-state structure and a modest energy barrier $(10 \mathrm{kcal} / \mathrm{mol}$ from the suitable conformation, $16 \mathrm{kcal} / \mathrm{mol}$ from the lowest-energy conformation of 1a-II) for the hydrogen atom migration to the nitrogen atom were found by DFT calculations. Compound 1a-Il protonated at one of the pyridazine nitrogen atoms was considered in the transition-state search. The hydrogen atom migration step is possible also for neutral compound 1a-II; however, the reaction barrier is higher by 18 $\mathrm{kcal} / \mathrm{mol}$ (see details in ESI). The computational study thus corroborates our presumption of both, the 'half-chair' conformation of 1a and its tendency to promote the formation of fluorescent 1,4-dihydropyridazines in reaction with 1,2,4,5tetrazines. For comparison, we also performed the transitionstate search for the hydrogen atom migration reaction for dioxolane fused TCO (d-TCO), which also contains a heteroatom and adopts the highly strained 'half-chair' conformation as reported previously. ${ }^{[9 \mathrm{~d}]}$ The calculated reaction barrier was found to be significantly higher $(28 \mathrm{kcal} / \mathrm{mol})$ than for compound 1a-II (See ESI) showing that it is the unique structure of aza-TCOs, which leads to the formation of fluorescent products by promoting the dihydropyridazine tautomerization. This unique behavior of aza-TCO was confirmed also experimentally (Fig. S5).

\section{Synthesis of aza-TCOs}

Encouraged by these computational results, we next focused on the synthesis of the aziridine-fused trans-cyclooctene. In our initial synthesis, we utilized Sharpless aziridination, ${ }^{[10]}$ which yielded the desired $\mathrm{N}$-tosyl-protected aziridine $\mathbf{3}$ in a single step (Scheme 1). Compound $\mathbf{3}$ was then conveniently converted to the corresponding trans-isomer $\mathbf{4}$ by the continuous flowphotoisomerization protocol developed by Fox and coworkers. ${ }^{[11]}$ Unfortunately, our attempts to efficiently remove the tosyl group using sodium naphthalenide ${ }^{[12]}$ were unsuccessful and led only to concomitant trans-to-cis isomerization of $(1 R, 8 S, E)$ 9-azabicyclo[6.1.0]non-4-ene 5. We therefore used a different strategy and started the synthesis from known (Z)-9-oxa bicyclo[6.1.0]non-4-ene 6. Opening of the epoxide afforded azido alcohol 7, which was converted to the corresponding aziridine 8 via the Staudinger reduction-cyclization reaction using triphenylphosphine. ${ }^{[13]}$ This sequence of reaction steps leads to cis-ring fusion which is known to induce the 'half-chair' conformation in bicyclononenes. ${ }^{[9 a, 9 c]}$ The next crucial step in the synthesis was the protection of the aziridine nitrogen atom. After extensive experimentation, we found that the trimethylsilylethoxycarbonyl (Teoc) protecting group enables efficient photoisomerization and subsequent mild deprotection to yield compound $\mathbf{5}$. Alkylation of the aziridine nitrogen afforded ethyl ester 11, which was reduced by $\mathrm{LiAlH}_{4}$ to the desired 2$((1 R, 8 S, E)-9$-azabicyclo[6.1.0]non-4-en-9-yl)ethan-1-ol 1a. Since the conformation of the trans-cyclooctene ring is crucial for its reactivity, we performed conformational analysis of compound 1a by NMR spectroscopy. All proton and carbon NMR signals were assigned by a combination of $1 \mathrm{D}$ and $2 \mathrm{D}$ NMR experiments and all observable proton-proton spin-spin coupling constants were determined. The experimental coupling constants are in excellent agreement with those calculated by using the DFT method for the lowest-energy (half-chair) 
A)

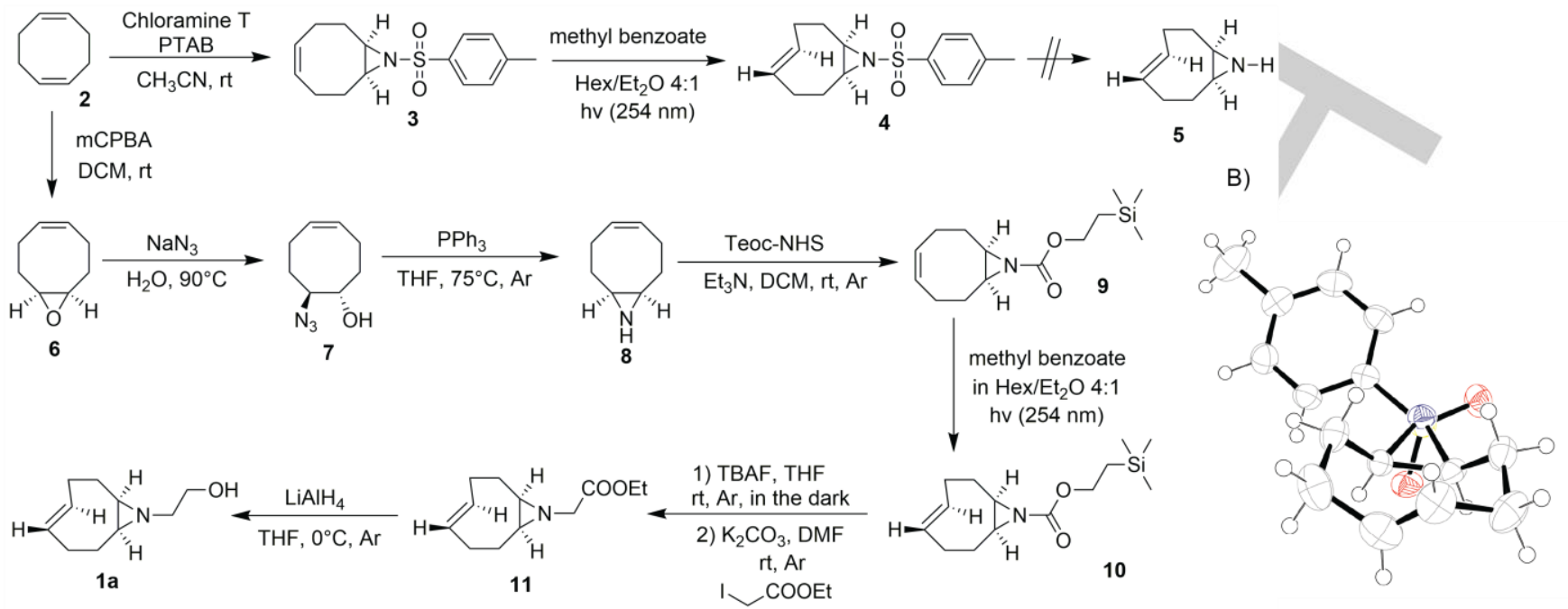

Scheme 1. A) Synthesis of aza-TCOs. B) An ORTEP view of the major enantiomer of compound 4 (displacement ellipsoids shown with $50 \%$ probability). CCDC 1553867.

conformer of 1a (Figure 1B). The 'half-chair' conformation of 1a was further confirmed by Nuclear Overhauser Effect Spectroscopy (NOESY); the most important NOE contacts for the determination of the conformation are depicted in Figure $1 \mathrm{~A}$. In addition, $\mathrm{N}$-tosylated bicyclononene $\mathbf{4}$ was a crystalline compound which afforded crystals suitable for X-Ray analysis and further confirmed the 'half-chair' conformation. The compound crystalized as a mixture of two enantiomers in a 6:4 ratio (Scheme 1B and Fig. S2).

\section{Reactivity of aza-TCOs and photophysical properties of the click products after reaction with 1,2,4,5-tetrazines}

As already predicted by the computations, the new aza-TCO dienophile 1a yields fluorescent products in the IEDDA reaction with tetrazines similarly to the previously reported ax-TCO-ol ${ }^{[8]} A$ series of experiments and calculations confirmed the identity of 1,4-dihydropyridazines as the actual fluorescent species formed during the reaction (see ESI). We determined the photophysical properties of the corresponding click products, which are summarized in Table 1. The dihydropyridazine products 13a-13e have large Stokes shifts and tunable emission maxima depending on the substitution pattern of the starting tetrazine (Fig. S7). The fluorescence enhancement upon the reaction ranges from 20- to an excellent 70 -fold. The fluorescence quantum yields are relatively low when compared to the more commonly used fluorophores (e.g. fluorescein $\phi_{\mathrm{fl}}=$ ca. 0.9 in 0.1 $\mathrm{M} \mathrm{NaOH})^{[14]}$ however, still sufficient for bioimaging applications as we show below.

The second order rate constants of the reaction between 1a and tetrazines 12a-12e were determined. The kinetic measurements were performed under pseudo first-order conditions using an excess of the dienophile. The decrease in concentration of the starting tetrazine was followed in time by measuring the decrease in its absorbance by UV-Vis spectroscopy. All measurements were carried out at least in triplicate and the average rate constants are summarized in Table 1 (for details see ESI). The second order rate constants range from $250 \mathrm{M}^{-1} \mathrm{~s}^{-1}$ to more than $6000 \mathrm{M}^{-1} \mathrm{~s}^{-1}$ (in $\mathrm{MeOH}$ at $21^{\circ} \mathrm{C}$ ), highlighting the exceptional reactivity of $1 \mathrm{a}$. The aza-TCO showed good reactivity toward 1,2,4-triazines as well, which were recently shown to be a good alternative to the more commonly used 1,2,4,5-tetrazines (Table S10). ${ }^{[15]}$

Furthermore, we used some of the known TCO derivatives to directly compare their reactivity with $\mathbf{1 a}$. We used diphenyl-stetrazine as the heterodiene in this case (Figure 2). Our data show, that 1 a reacts about 20 times faster than simple ax-TCOol (Table S9), which we have used previously in fluorogenic cell labeling experiments. ${ }^{[8]}$

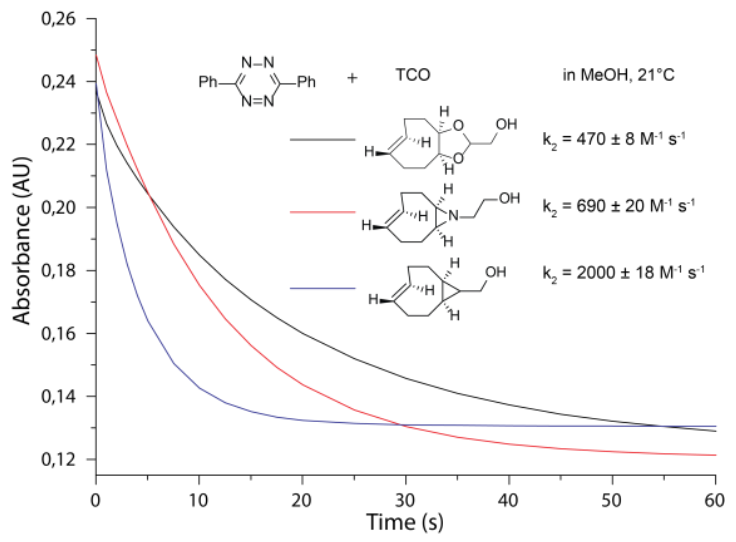

Figure 2. Comparison of the reactivity of various TCOs in reaction with diphenyl-s-tetrazine measured in $\mathrm{MeOH}$ at $21^{\circ} \mathrm{C}$ using manual stopped-flow device connected to UV-Vis spectrophotometer. 
Table 1. Photophysical properties of click products and second-order rate constants of the reaction between 1,2,4,5-tetrazines and aza-TCO 1a.

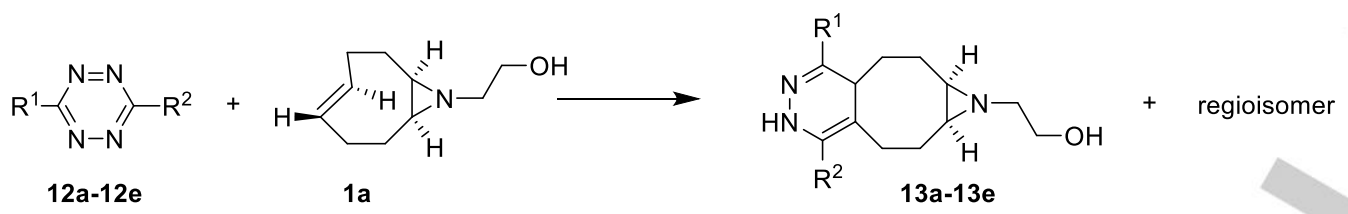

\begin{tabular}{|c|c|c|c|c|c|c|c|c|c|c|}
\hline Entry & Tetrazine & $\begin{array}{l}\text { Click } \\
\text { product }\end{array}$ & $\mathrm{R}^{1}$ & $\mathrm{R}^{2}$ & $\begin{array}{l}\text { Fluorescence of } \\
\text { the click product }\end{array}$ & $\begin{array}{l}\lambda_{\mathrm{Abs}} / \\
\lambda_{\mathrm{Em}}{ }^{[b]}\end{array}$ & $\begin{array}{l}\text { Stokes } \\
\text { shift }^{[b]}\end{array}$ & $\phi_{\mathrm{fl}} / \mathcal{E}_{\max }^{[\mathrm{cc}]}$ & $\begin{array}{l}\text { Intensity } \\
\text { increase }\end{array}$ & $\left(M^{-1} s^{-1}\right)^{[d]}$ \\
\hline 1 & $12 a$ & $13 a$ & & & - & $317 / 478$ & 161 & $0.06 / 12$ & 20 -fold & $6360 \pm 90$ \\
\hline 2 & $12 b$ & $13 b$ & & & & $333 / 5$ & 168 & $0.07 / 21$ & 60 -fold & $318 \pm 3$ \\
\hline 3 & $12 c$ & $13 c$ & & & & $321 / 475$ & 154 & $0.15 / 16$ & 55 -fold & $255 \pm 7$ \\
\hline 4 & $12 d$ & $13 d$ & & & & $337 / 540$ & 203 & $0.04 / 23$ & 40 -fold & $316 \pm 2$ \\
\hline 5 & $12 e$ & $13 e$ & & & & $335 / 489$ & 154 & $0.11 / 17$ & 70 -fold & $740 \pm 25$ \\
\hline
\end{tabular}

[a] Pictures were captured in quartz cuvettes using a hand-held UV lamp ( $365 \mathrm{~nm}$ ), [b] given in $\mathrm{nm}$, [c] extinction coefficients $\mathcal{E}_{\text {max }}$ are in $10^{3} \mathrm{M}^{-1} \mathrm{~cm}^{-1}$, quantum yields were determined using quinine sulfate in $0.5 \mathrm{M} \mathrm{H}_{2} \mathrm{SO}_{4}$ as standard, [d] kinetic measurements were performed in $\mathrm{MeOH}$ at room temperature under pseudo first-order conditions using an excess of $\mathbf{1 a}$ (for details see ESI).

Compound 1a reacts about 1.5 times faster than the dioxolanefused TCO (d-TCO) reported by Fox and coworkers. ${ }^{[9 \mathrm{~d}]}$ The only derivative, which exceeded 1a in reactivity (about 3 times), was the cyclopropyl-fused trans-cyclooctene (s-TCO) developed by the same group. ${ }^{[9]}$ These data clearly show that fusion of an aziridine ring to trans-cyclooctenes yields highly reactive dienophiles belonging to the fastest reacting bioorthogonal probes known to date.

\section{Stability of aza-TCOs and of the click products under biologically relevant conditions}

We then studied the stability of the newly developed dienophile under various conditions. Compound 1a showed excellent stability when stored in the dark at room temperature as a solution in $\mathrm{CD}_{3} \mathrm{OD}$ (Fig. S9). The stability of the compound in the presence of L-cysteine, which is known to promote the trans-to-cis isomerization of TCOs, ${ }^{[9 d, 16]}$ was also very good. A 1:1 mixture of cis and trans isomers was obtained in 7 days after subjecting compound $\mathbf{1 a}$ to 3.5 equivalents of L-cysteine at neutral $\mathrm{pH}$ in deuterated phosphate buffer (Fig. S10). We also examined the stability of the click products. The 1,4dihydropyridazines formed upon reaction of 1a with diphenyl-stetrazine or dipyridyl-s-tetrazine are stable under various conditions (e.g. phosphate buffered saline, fetal bovine serum) for several days. HPLC-MS analysis showed that the corresponding fully aromatic pyridazines are formed as major 'side' products in this case. Our data show that the click product of diphenyl-s-tetrazine oxidizes faster than the click product of dipyridyl-s-tetrazine (Fig. S11).

\section{Fluorogenic labeling of peptides}

To evaluate the performance of $\mathbf{1 a}$ as a bioorthogonal handle for fluorogenic biomolecule tagging, we first performed labeling experiments on a model peptide. Our attempts to prepare the active ester directly from 1a failed, giving under various reaction conditions a mixture of products.

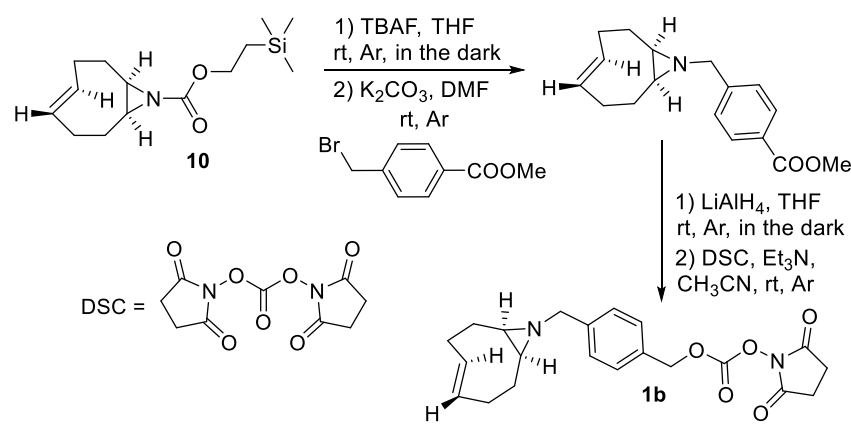

Scheme 2. Synthesis of aza-TCO active ester $\mathbf{1 b}$. 


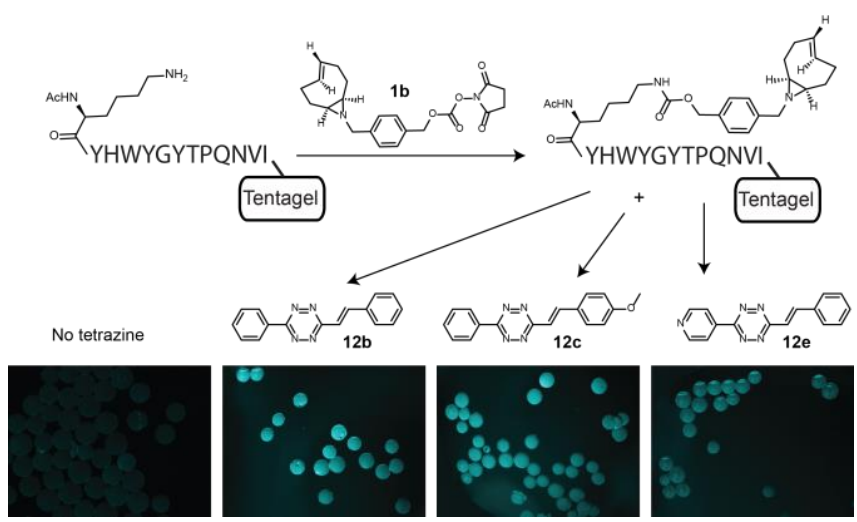

Figure 3. The fluorogenic click reaction was performed on a model peptide modified with aza-TCO 1b using various tetrazine probes. Pictures were captured under a fluorescence stereomicroscope at $350 \mathrm{~nm}$ irradiation using CoolLED pE-300 light source and the following setup in each case: Exposure time $300 \mathrm{~ms}$, IL light intensity 1000 and objective $1 \times / 0.08$. Colors were finally adjusted using LAS AF Lite program.

Similarly, the hydrolysis of ethyl ester $\mathbf{1 1}$ also did not afford the desired carboxylic acid derivative. Fortunately, the structural features of aza-bicyclononene $\mathbf{5}$ enable a straightforward synthesis of its derivatives by simple alkylation of the free aziridine nitrogen atom. After optimization, we prepared the active ester of aza-TCO using bromomethyl benzoate as the alkylating agent followed by reduction of the ester to the corresponding alcohol and finally by reaction with $N, N^{\prime}$ disuccinimidyl carbonate (Scheme 2 and ESI). Active ester 1b was first reacted with the model peptide (KYHWYGYTPQNVI) containing an N-terminal lysine. The final aza-TCO-modified peptide, still attached to the resin (Tentagel-OH), was treated with various tetrazines $(\mathbf{1 2 b}, \mathbf{1 2 c}$ and $\mathbf{1 2 e})$ and the formation of fluorescent products was verified using fluorescence microscopy (Figure 3 and Fig. S12). We observed the successful formation of the desired click products in all cases, which was also confirmed by HPLC-MS analysis (Fig. S13-S17). Our previous experiments with the ax-TCO-ol showed that the fluorogenic properties of the reaction under these conditions alter or even completely disappear. ${ }^{[8]}$ Aza-TCOs are therefore superior compounds for fluorescent peptide labeling applications.

\section{Fluorogenic labeling of proteins}

The active ester-mediated protein modification still belongs to the most frequently applied methodology for covalent protein modification. ${ }^{[17]}$ Alternatively, a cysteine-maleimide reaction can be used for this purpose. ${ }^{[18]}$ We performed a series of experiments to probe aza-TCO derivatives for the fluorogenic labeling of proteins. We first modified human carbonic anhydrase II (hCAll) with the active ester $\mathbf{1 b}$ under standard conditions and added tetrazine 12c to the protein (Figure 4 and Fig. S18). We also used a biotin-conjugated tetrazine probe 12f, which enables double detection after the reaction. Namely by spectrophotometry, based on the formation of the fluorescent dihydropyridazine, and by western blot analysis due to the presence of the biotin moiety. SDS-PAGE analysis of the reaction mixtures showed that the reaction proceeded efficiently and the fluorescently-labeled products were clearly visible after UV irradiation of the gel (Figure 4A). The presence of the biotin was successfully confirmed by western blot analysis using fluorescein-conjugated streptavidin. The fluorogenicity, which is an intrinsic property of the chemical reaction, thus facilitates straightforward detection of the reaction products and should enable the development of e.g. multimodal detection probes similar to $\mathbf{1 2 f}$.

We then used ADP-ribosylation factor 1 protein (Arf1), which was prepared using standard recombinant techniques. ${ }^{[19]}$ Arf1 contains a single cysteine residue, which was used for the attachment of aza-TCO using maleimide 1c. The success of the reaction after addition of $12 \mathrm{c}$ was evident from the shift of the protein band on SDS-PAGE gel as well as after spectrophotometric imaging (Figure 4B and Fig. S19). These experiments manifest that aza-TCOs are useful bioconjugation reagents enabling efficient fluorogenic tagging of proteins.

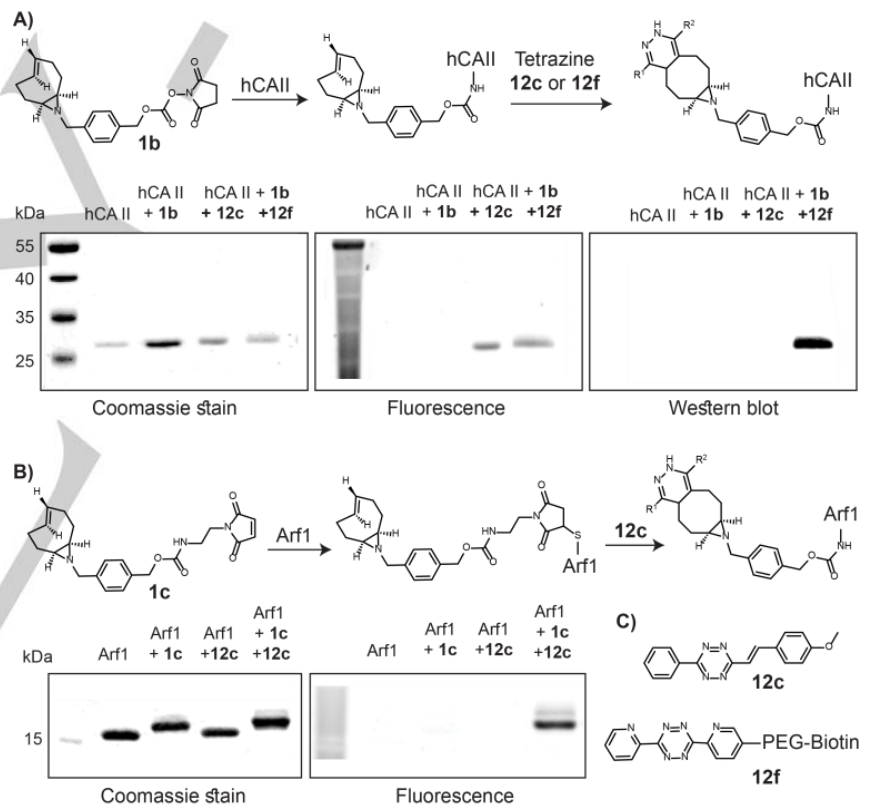

Figure 4. Fluorogenic labeling of proteins. A) Labeling of aza-TCO-modified hCAll using tetrazine 12c and 12f, B) labeling of Arf1 protein via maleimide and subsequent reaction with tetrazine $\mathbf{1 2 c}$.

\section{Fluorogenic labeling of glycoconjugates on U2OS cancer cells}

We next turned our attention to cell labeling experiments to further demonstrate the potential of aza-TCOs for bioimaging applications. First, we attached the aza-TCO moiety to concanavalin $A$ (ConA). ConA is a lectin which shows high specificity for $\alpha$-D-mannose- and $\alpha$-D-glucose-containing glycoconjugates and is useful in carbohydrate studies. ${ }^{[20]}$ To compare the performance of aza-TCO with the previously reported ax-TCO-ol, we also conjugated two different axial 
TCOs to ConA. Derivative $\mathbf{1 d}$ is attached to ConA via a carbamate linker while $1 e$ via a peptide bond. We next separately added the three TCO-modified ConAs to fixed and permeabilized U2OS cancer cells to enable intracellular glycoconjugate staining after addition of 12c.
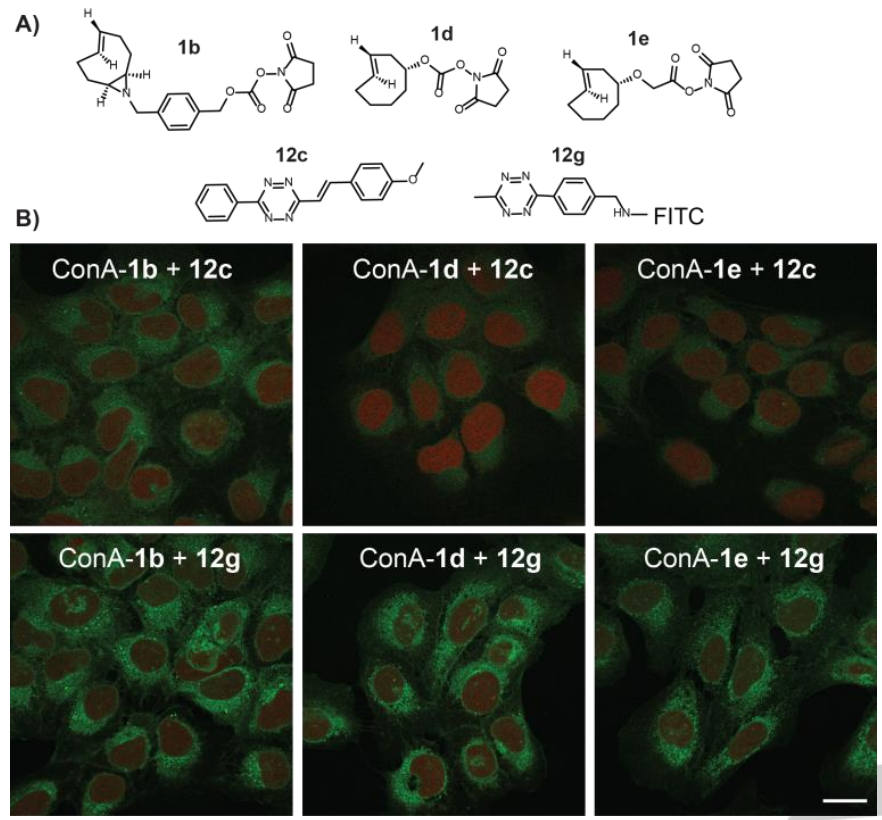

Figure 5. A) Structures of active esters used to modify ConA and structure of tetrazines $\mathbf{1 2 c}$ and $\mathbf{1 2 g}$ used for labeling. B) Confocal microscope images of fluorogenic U2OS cell labeling using ConA modified with TCOs $\mathbf{1 b}, \mathbf{1 d}, \mathbf{1 e}$ and tetrazine 12c or FITC-conjugated tetrazine $\mathbf{1 2 g}$. Conditions: The cells were fixed, permeabilized and incubated for 1 hour with the respective TCO modified ConA $(0.25 \mu \mathrm{g} / \mu \mathrm{L})$. After washing, the cells were incubated with 0.25 $\mathrm{mM}$ tetrazine and $5 \mu \mathrm{M}$ DRAQ5 for $10 \mathrm{~min}$ and imaged under confocal microscope. Click product (ex.: $405 \mathrm{~nm}$, em.: 560-666 nm); FITC channel (ex.: $458 \mathrm{~nm}$, em.: 490-552 nm); the nucleus was stained with DRAQ5 (ex.: $633 \mathrm{~nm}$ em.: 653-732 nm). Scale bar: $20 \mu \mathrm{m}$.

We used fluorescein-tagged tetrazine $\mathbf{1 2 g}$ as a positive control in this experiment as this compound yields fluorescent products in each case. The formation of fluorescent products was verified by inspecting the cells under a confocal microscope (Figure 5 and Fig. S21). Our experiment confirmed the superior performance of the newly developed aza-TCO. ConA modified with $\mathbf{1 b}$ gave the most intensive labeling when compared to ConA modified with either $\mathbf{1 d}$ or $\mathbf{1 e}$, although a weak staining of glycoconjugates was visible in these cases as well. On the other hand, equal staining of the cells was observed when we used FITC-tetrazine derivative $\mathbf{1 2 g}$ under the same conditions (Figure $5 \mathrm{~B}$ and Fig. S21).

\section{Fluorogenic imaging of peptidoglycans in live bacteria}

Various derivatives of $\mathrm{D}$-amino acids can be incorporated into the peptidoglycans (PGs) of diverse bacterial species and can be used as valuable chemical probes providing information about dynamics, synthesis and maturation of PGs. ${ }^{[21]}$ In order to test if our aza-TCOs could serve as a reporter molecule for similar application, we prepared the aza-TCO modified D-amino acid 1f (See ESI). The gram-positive Bacillus subtilis were first grown in the presence of $\mathbf{1 f}$ for 2 hours. Similarly, gram-negative E. coli cells were grown in the presence of $\mathbf{1 f}$ for different period of time (2, 6 or 12 hours). Incorporation of the amino acid into PGs was subsequently validated under confocal microscope after labeling with the fluorogenic BODIPY-tetrazine probe $\mathbf{1 2}$. We observed a fluorescent signal on the surface of the bacteria after incubation with $1 \mathrm{f}$ and labeling with $\mathbf{1 2 h}$, while no fluorescence formed in cells incubated only with the amino acid 1f or tetrazine 12h under the same conditions (Figure 6B-C and Fig. S22-23). Weak fluorescent signal formed in E. coli cells even after 12 hours of incubation with $\mathbf{1 f}$ and subsequent labeling with $12 \mathrm{~h}$ showing that at least part of the modified amino acid remains in the highly reactive trans-conformation within this time period. These experiments further demonstrate that the aza-TCO moiety can serve as valuable reporter group for various bioimaging applications. The high reactivity accompanied with good stability favors aza-TCOs for experiments where both of these attributes are important and desirable.

A)

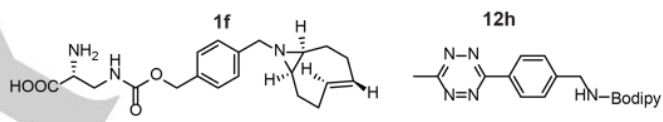

B)

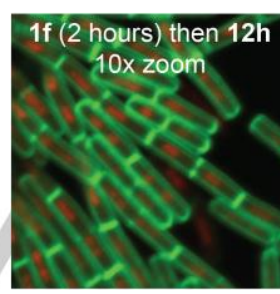

C)

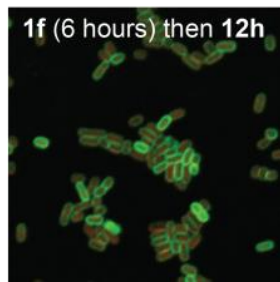

Labeling of B. subtilis

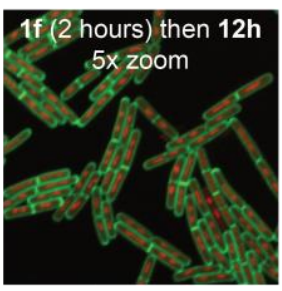

Labeling of E. coli

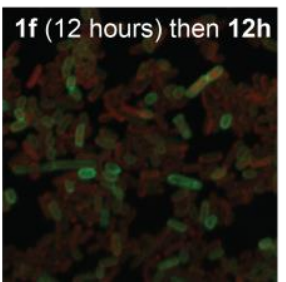

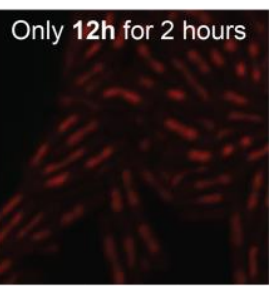

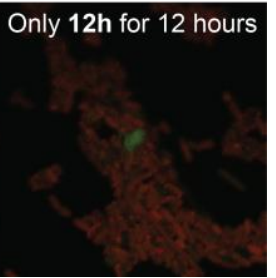

Figure 6. A) Structure of D-amino acid $1 \mathrm{f}$ and Tetrazine-bodipy 12h. B) and C) Confocal microscope images of fluorogenic bacterial cell wall labeling using $\mathbf{1 f}$ and 12h. Conditions: Live B. subtilis or E. coli cells were incubated with 1f (1 $\mathrm{mM})$ for the indicated time. After washing, the cells were incubated for $15 \mathrm{~min}$ with $12 \mathrm{~h}(25 \mu \mathrm{M})$ and $50 \mu \mathrm{M}$ DRAQ5. The cell were then inspected under confocal microscope using the following set up: nucleus was stained with DRAQ5 (ex.: $633 \mathrm{~nm}$, em.: 653-732 nm) and click product resulting from the reaction (ex.: $458 \mathrm{~nm}$, em.: 499-569 nm). $5 x$ and $10 x$ zoom for $B$. subtilis, 10x zoom for E.coli.

\section{Conclusions}


In this study, we present the design and synthesis of aziridinefused trans-cyclooctene derivatives, which produce fluorescent products in reaction with 1,2,4,5-tetrazines. The newly developed dienophiles adopt the 'half-chair' conformation as a result of the cis-ring fusion of the aziridine moiety. This highly strained conformation leads to remarkable reactivity in the IEDDA reactions making these compounds one of the most reactive bioorthogonal probes known to date. Despite their high reactivity, these compounds show good stability under various conditions. Stability of the corresponding click products was found to be excellent as well. We demonstrate the potential of aza-TCOs in fluorogenic ligation reactions by the labeling of model biomolecules and by cell imaging experiments. The exceptional reactivity together with the intrinsic formation of fluorescent products upon reaction with simple tetrazines make aza-TCO dienophiles attractive candidates for fluorogenic biomolecule tagging and bioimaging applications.

\section{Acknowledgements}

This work was supported by the Czech Science Foundation (P207/15-06020Y, for M.V.), Ministry of Education Youth and Sports of the Czech Republic (project LO1304, program "NPUI") and by the European Research Council (ERC) under the European Union's Horizon 2020 research and innovation programme (grants agreement No 677465 , for M.V.). We thank A. M. Downey for critical reading of the manuscript. We acknowledge the kind donation of hCAll (P. Řezáčová), Arf1 (D. Chalupská) used in model protein labeling experiments and B.subtilis provided by K. Stříšovský lab.

\section{Conflict of interest}

The authors declare no conflict of interest.

Keywords: cell labeling • Diels-Alder reaction • fluorogenic • trans-cyclooctene $\cdot$ tetrazines

[1] a) A. Nadler, C. Schultz, Angew. Chem. Int. Ed. 2013, 52, 2408-2410; b) G. B. Cserép, A. Herner, P. Kele, Methods Appl. Fluoresc. 2015, 3 042001.

[2] a) M. J. Hangauer, C. R. Bertozzi, Angew. Chem. Int. Ed. 2008, 47, 2394-2397; b) G. A. Lemieux, C. L. De Graffenried, C. R. Bertozzi, J. Am. Chem. Soc. 2003, 125, 4708-4709.

[3] a) F. Friscourt, C. J. Fahrni, G. J. Boons, J. Am. Chem. Soc. 2012, 134, 18809-18815; b) A. Herner, I. Nikic, M. Kallay, E. A. Lemke, P. Kele, Org. Biomol. Chem. 2013, 11, 3297-3306; c) C. Le Droumaguet, C. Wang, Q. Wang, Chem. Soc. Rev. 2010, 39, 1233-1239; d) J. Li, M. Hu, S. Q. Yao, Org. Lett. 2009, 11, 3008-3011; e) J. Qi, M. S. Han, Y. C. Chang, C. H. Tung, Bioconjugate Chem. 2011, 22, 1758-1762; f) P. Shieh, M. S. Siegrist, A. J. Cullen, C. R. Bertozzi, Proc. Natl. Acad. Sci. 2014, 111, 5456-5461; g) K. Sivakumar, F. Xie, B. M. Cash, S. Long, H. N. Barnhill, Q. Wang, Org. Lett. 2004, 6, 4603-4606; h) Z. Zhou, C. J. Fahrni, J. Am. Chem. Soc. 2004, 126, 8862-8863; i) O. Demeter, A.
Kormos, C. Koehler, G. Mező, K. Németh, E. Kozma, L. B. Takács, E. A. Lemke, P. Kele, Bioconjugate Chem. 2017, 28, 1552-1559.

[4] a) Y. Wang, C. I. Vera, Q. Lin, Org. Lett. 2007, 9, 4155-4158; b) W Song, Y. Wang, J. Qu, Q. Lin, J. Am. Chem. Soc. 2008, 130, 9654 9655; c) W. Song, Y. Wang, J. Qu, M. M. Madden, Q. Lin, Angew. Chem. Int. Ed. 2008, 47, 2832-2835; d) Z. Yu, L. Y. Ho, Q. Lin, J. Am Chem. Soc. 2011, 133, 11912-11915; e) E. Kaya, M. Vrabel, C. Deiml, S. Prill, V. S. Fluxa, T. Carell, Angew. Chem. Int. Ed. 2012, 51, 4466 4469; f) Z. Yu, T. Y. Ohulchanskyy, P. An, P. N. Prasad, Q. Lin, J. Am. Chem. Soc. 2013, 135, 16766-16769; g) X. S. Wang, Y. J. Lee, W. R Liu, Chem. Commun. 2014, 50, 3176-3179.

[5] a) N. K. Devaraj, S. Hilderbrand, R. Upadhyay, R. Mazitschek, R. Weissleder, Angew. Chem. Int. Ed. 2010, 49, 2869-2872; b) J. Yang, J. Seckute, C. M. Cole, N. K. Devaraj, Angew. Chem. Int. Ed. 2012, 51, 7476-7479; c) L. G. Meimetis, J. C. Carlson, R. J. Giedt, R. H. Kohler, R. Weissleder, Angew. Chem. Int. Ed. 2014, 53, 7531-7534; d) A Wieczorek, T. Buckup, R. Wombacher, Org. Biomol. Chem. 2014, 12, 4177-4185; e) H. Wu, J. Yang, J. Seckute, N. K. Devaraj, Angew. Chem. Int. Ed. 2014, 53, 5805-5809; f) E. Kozma, O. Demeter, P. Kele, ChemBioChem 2017, 18, 486-501; g) A. Wieczorek, P. Werther, J. Euchner, R. Wombacher, Chem. Sci. 2017, 8, 1506-1510; h) E. Kozma, G. Estrada Girona, G. Paci, E. A. Lemke, P. Kele, Chem. Commun. 2017, 53, 6696-6699.

[6] C. P. Ramil, Q. Lin, Curr. Opin. Chem. Biol. 2014, 21, 89-95.

[7] X. Shang, X. Song, C. Faller, R. Lai, H. Li, R. Cerny, W. Niu, J. Guo, Chem. Sci. 2017, 8, 1141-1145.

[8] A. Vazquez, R. Dzijak, M. Dracinsky, R. Rampmaier, S. J. Siegl, M. Vrabel, Angew. Chem. Int. Ed. 2017, 56, 1334-1337.

[9] a) R. D. Bach, J. Am. Chem. Soc. 2009, 131, 5233-5243; b) J. Dommerholt, S. Schmidt, R. Temming, L. J. Hendriks, F. P. Rutjes, J. C. van Hest, D. J. Lefeber, P. Friedl, F. L. van Delft, Angew. Chem. Int. Ed. 2010, 49, 9422-9425; c) M. T. Taylor, M. L. Blackman, O. Dmitrenko, J. M. Fox, J. Am. Chem. Soc. 2011, 133, 9646-9649; d) A. Darko, S Wallace, O. Dmitrenko, M. M. Machovina, R. A. Mehl, J. W. Chin, J. M. Fox, Chem. Sci. 2014, 5, 3770-3776.

[10] J. U. Jeong, B. Tao, I. Sagasser, H. Henniges, K. B. Sharpless, J. Am. Chem. Soc. 1998, 120, 6844-6845.

[11] M. Royzen, G. P. Yap, J. M. Fox, J. Am. Chem. Soc. 2008, 130, 3760 3761.

[12] S. C. Bergmeier, P. P. Seth, Tetrahedron Lett. 1999, 40, 6181-6184.

[13] Y. Ittah, Y. Sasson, I. Shahak, S. Tsaroom, J. Blum, J. Org. Chem 1978, 43, 4271-4273.

[14] A. M. Brouwer, Pure Appl. Chem. 2011, 83, 2213-2228.

[15] a) K. A. Horner, N. M. Valette, M. E. Webb, Chem. Eur. J. 2015, 21, 14376-14381; b) D. N. Kamber, Y. Liang, R. J. Blizzard, F. Liu, R. A Mehl, K. N. Houk, J. A. Prescher, J. Am. Chem. Soc. 2015, 137, 83888391; c) S. J. Siegl, R. Dzijak, A. Vazquez, R. Pohl, M. Vrabel, Chem. Sci. 2017, 8, 3593-3598.

[16] R. Rossin, S. M. van den Bosch, W. Ten Hoeve, M. Carvelli, R. M Versteegen, J. Lub, M. S. Robillard, Bioconjugate Chem. 2013, 24, 1210-1217.

[17] a) E. Basle, N. Joubert, M. Pucheault, Chem. Biol. 2010, 17, 213-227; b) O. Koniev, A. Wagner, Chem. Soc. Rev. 2015, 44, 5495-5551.

[18] S. B. Gunnoo, A. Madder, ChemBioChem 2016, 17, 529-553.

[19] A. Baumlova, D. Chalupska, B. Róźycki, M. Jovic, E. Wisniewski, M. Klima, A. Dubankova, D. P. Kloer, R. Nencka, T. Balla, E. Boura EMBO Rep. 2014, 15, 1085-1092.

[20] I. J. Goldstein, R. D. Poretz, in The Lectins. Properties, Functions, and Applications in Biology and Medicine. , Vol. 32 (Eds.: I. E. Liener, N. Sharon, I. J. Goldstein), Elsevier, 1986, pp. 33-247.

[21] a) M. S. Siegrist, S. Whiteside, J. C. Jewett, A. Aditham, F. Cava, C. R. Bertozzi, ACS Chem. Biol. 2013, 8, 500-505; b) E. Kuru, S. Tekkam, E. Hall, Y. V. Brun, M. S. Van Nieuwenhze, Nat. Protoc. 2015, 10, 33-52. 


\section{FULL PAPER}

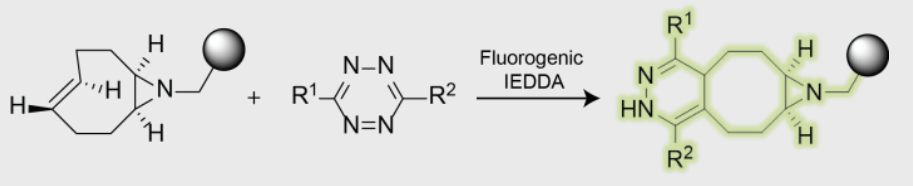

Super-reactive dienophiles: Fusion of an aziridine ring to trans-cyclooctenes was found to enhance their reactivity while still maintaining good stability under biological conditions. These aza-TCOs lead to the formation of fluorescent products upon reaction with 1,2,4,5-tetrazines which is useful for bioimaging applications.
Sebastian J. Siegl, Arcadio Vázquez,

Rastislav Dzijak, Martin Dračínský, Juraj Galeta, Robert Rampmaier, Blanka Klepetárová, and Milan Vrabel*

Page No. - Page No.

Design and synthesis of azabicyclononene dienophiles for rapid fluorogenic ligations 